\title{
Numerical Solution of a Class of Functional-Differential Equations Using Jacobi Pseudospectral Method
}

\author{
A. H. Bhrawy, ${ }^{1,2}$ M. A. Alghamdi, ${ }^{1}$ and D. Baleanu ${ }^{3,4,5}$ \\ ${ }^{1}$ Department of Mathematics, Faculty of Science, King Abdulaziz University, Jeddah 21589, Saudi Arabia \\ ${ }^{2}$ Department of Mathematics, Faculty of Science, Beni-Suef University, Beni-Suef 62511, Egypt \\ ${ }^{3}$ Department of Chemical and Materials Engineering, Faculty of Engineering, King Abdulaziz University, Jeddah 21589, Saudi Arabia \\ ${ }^{4}$ Department of Mathematics and Computer Sciences, Cankaya University, Eskisehir Yolu 29.km, 06810 Ankara, Turkey \\ ${ }^{5}$ Institute of Space Sciences, P.O. Box MG-23, 76900 Magurele-Bucharest, Romania
}

Correspondence should be addressed to A. H. Bhrawy; alibhrawy@yahoo.co.uk

Received 24 August 2013; Accepted 18 September 2013

Academic Editor: Soheil Salahshour

Copyright (C) 2013 A. H. Bhrawy et al. This is an open access article distributed under the Creative Commons Attribution License, which permits unrestricted use, distribution, and reproduction in any medium, provided the original work is properly cited.

The shifted Jacobi-Gauss-Lobatto pseudospectral (SJGLP) method is applied to neutral functional-differential equations (NFDEs) with proportional delays. The proposed approximation is based on shifted Jacobi collocation approximation with the nodes of Gauss-Lobatto quadrature. The shifted Legendre-Gauss-Lobatto Pseudo-spectral and Chebyshev-Gauss-Lobatto Pseudo-spectral methods can be obtained as special cases of the underlying method. Moreover, the SJGLP method is extended to numerically approximate the nonlinear high-order NFDE with proportional delay. Some examples are displayed for implicit and explicit forms of NFDEs to demonstrate the computation accuracy of the proposed method. We also compare the performance of the method with variational iteration method, one-leg $\theta$-method, continuous Runge-Kutta method, and reproducing kernel Hilbert space method.

\section{Introduction}

In the last four decades, spectral method has become increasingly popular and been successfully applied in solving all types of differential equations owing to its high order of accuracy (see, for instance, [1-5]). Recently there has been a growing interest in applying spectral methods for the numerical solution of fractional differential equations and delay differential equations (DDEs). Moreover, the principal difficulty in studying NFDEs with proportional delays lies in their special transcendental nature. Thus we propose an efficient technique to solve such differential equations numerically based on shifted Jacobi polynomials.

In the last two decades, some numerical approaches for treating several types of DDEs were presented in [6-12]. In this direction, Zhao et al. [13] studied the stability of RungeKutta approach and applied it for solving the NFDEs with proportional delays. Tohidi et al. [12] derived the operational matrix of Bernoulli polynomial and used it with collocation method to solve the pantograph type equation. The one-leg $\theta$ approach was implemented to solve nonlinear NFDEs in [14]. Meanwhile, Trif [15] proposed a direct solution technique by using the Chebyshev Tau operational matrix method for solving the pantograph type equation. More recently, Bhrawy et al. [16] proposed the Legendre pseudospectral algorithm with studying the error analysis and stability of the proposed algorithm for a class of DDEs. Sun and Zhang [17] proposed a compact difference method for solving nonlinear partial DDE. Cordero and Escalante [18] extended the application of segmented tau approach for solving a class of NFDEs, meanwhile, the history-valued NFDEs were approximated in [19]. Işik et al. [20] introduced a new Bernstein collocation method for the numerical solution of DDEs of pantographtype with retarded case. The existence of solutions of neutral functional-differential equations with proportional delays had been discussed in [21-24].

The aim of this paper is to develop a direct solution technique to approximate the linear high-order NFDEs with 
proportional delays using the shifted Jacobi polynomials on the interval $[0, L]$; we dedicate the shifted Jacobi-GaussLobatto pseudospectral (SJGLP) method to find the approximate solution $u_{N}(x)$. Approximate semianalytical solution with high accuracy can be obtained by selecting a limited number of Gauss-Lobatto collocation points for the linear and nonlinear high-order NFDEs with proportional delays. For suitable collocation points we use the $(N-m+1)$ nodes of the shifted Jacobi-Gauss-Lobatto interpolation on $(0, L)$ in which the nodes are distinct and lie between 0 and $L$, and the two endpoints of the domain ( 0 and $L)$ are used as the first and last collocation points, respectively. The algorithms given in [16] can be obtained as special cases from the proposed algorithms. Finally, the accuracy of the proposed method is showed by test problems. From the results, these algorithms are extremely efficient and accurate for solving NFDEs.

The paper is arranged in the following way. In the next section, some basic properties of Jacobi polynomials which are required in the present paper are given, and in Section 3, the way of constructing the pseudospectral technique for NFDEs with proportional delays is described using the shifted Jacobi polynomials. In Section 4, we investigate the shifted Jacobi-Gauss-Lobatto pseudo-spectral (SJGLP) method for solving nonlinear high-order NFDEs with proportional delays. Some numerical results exhibiting the accuracy of the proposed algorithm are given in Section 5. Finally, a conclusion is given in Section 6.

\section{Preliminaries}

In this section, we briefly recall some properties of the Jacobi polynomials $\left(J_{k}^{(\theta, 9)}(x), k=0,1, \ldots, \theta>-1, \vartheta>-1\right)$, which satisfy the following relations:

$$
\begin{gathered}
J_{k}^{(\theta, 9)}(-x)=(-1)^{k} J_{k}^{(\theta, \vartheta)}(x), \\
J_{k}^{(\theta, 9)}(-1)=\frac{(-1)^{k} \Gamma(k+\vartheta+1)}{k ! \Gamma(\vartheta+1)} \\
J_{k}^{(\theta, \vartheta)}(1)=\frac{\Gamma(k+\theta+1)}{k ! \Gamma(\theta+1)} .
\end{gathered}
$$

The $q$ th derivative of Jacobi polynomials of degree $k$ can be given by

$$
D^{(q)} J_{k}^{(\theta, 9)}(x)=\frac{\Gamma(j+\theta+\vartheta+q+1)}{2^{q} \Gamma(j+\theta+\vartheta+1)} J_{k-q}^{(\theta+q, \vartheta+q)}(x) .
$$

These polynomials are the only polynomials arising as eigenfunctions of the following singular Sturm-Liouville equation:

$$
\begin{gathered}
\left(1-x^{2}\right) \phi^{\prime \prime}(x)+[\vartheta-\theta+(\theta+\vartheta+2) x] \phi^{\prime}(x) \\
+n(n+\theta+\vartheta+1) \phi(x)=0 .
\end{gathered}
$$

Let $w^{(\theta, 9)}(x)=(1-x)^{\theta}(1+x)^{\vartheta}$; then we define the weighted space $L_{w^{(\theta, 9)}}^{2}$ as usual. The inner product and the norm of
$L_{w^{(\theta, 9)}}^{2}$ with respect to the weight function are defined as follows:

$$
\begin{gathered}
(u, v)_{w^{(\theta, 9)}}=\int_{-1}^{1} u(x) v(x) w^{(\theta, 9)}(x) d x, \\
\|u\|_{w^{(\theta, 9)}}=(u, u)_{w^{(\theta, 9)}}^{1 / 2}
\end{gathered}
$$

The set of Jacobi polynomials forms a complete $L_{w^{(\theta, 9)}}^{2}$ orthogonal system, and

$$
\begin{aligned}
\left\|J_{k}^{(\theta, \vartheta)}\right\|_{w^{(\theta, 9)}} & :=h_{k} \\
& =\frac{2^{\theta+\vartheta+1} \Gamma(k+\theta+1) \Gamma(k+\vartheta+1)}{(2 k+\theta+\vartheta+1) \Gamma(k+1) \Gamma(k+\theta+\vartheta+1)} .
\end{aligned}
$$

Let us denote $P_{L, k}^{(\alpha, \beta)}(x)=J_{k}^{(\alpha, \beta)}((2 x / L)-1), L>0$. By the shifted Jacobi polynomial of degree $k$ and by using (1) and (2); then we deduce that

$$
\begin{gathered}
P_{L, k}^{(\alpha, \beta)}(0)=(-1)^{k} \frac{\Gamma(k+\beta+1)}{\Gamma(\beta+1) k !}, \\
D^{q} P_{L, k}^{(\alpha, \beta)}(0)=\frac{(-1)^{k-q} \Gamma(k+\beta+1)(k+\alpha+\beta+1)_{q}}{L^{q} \Gamma(k-q+1) \Gamma(q+\beta+1)}, \\
D^{m} P_{L, k}^{(\alpha, \beta)}(x)=\frac{\Gamma(m+k+\alpha+\beta+1)}{L^{m} \Gamma(k+\alpha+\beta+1)} P_{L, k-m}^{(\alpha+m, \beta+m)}(x) .
\end{gathered}
$$

The following inner product and norm

$$
\begin{gathered}
(u, v)_{w_{L}^{(\alpha, \beta)}}=\int_{0}^{L} u(x) v(x) w_{L}^{(\alpha, \beta)}(x) d x, \\
\|v\|_{w_{L}^{(\alpha, \beta)}}=(v, v)_{w_{L}^{(\alpha, \beta)}}^{1 / 2},
\end{gathered}
$$

are defined on the weighted space $L_{w_{L}^{(\alpha, \beta)}}^{2}(0, L)$ subject to the weight function $w_{L}^{(\alpha, \beta)}(x)=(L-x)^{\alpha} x^{\beta}$. Moreover, $P_{L, k}^{(\alpha, \beta)}(x)$ forms a complete $L_{w_{L}^{(\alpha, \beta)}}^{2}(0, L)$-orthogonal system.

According to (5), we get

$$
\left\|P_{L, k}^{(\alpha, \beta)}\right\|_{w_{L}^{(\alpha, \beta)}}^{2}=\left(\frac{L}{2}\right)^{\alpha+\beta+1} h_{k}^{(\alpha, \beta)}:=h_{L, k}^{(\alpha, \beta)} .
$$

\section{Linear High-Order NFDE with Proportional Delay}

In this section, we shall investigate solutions to NFDEs with proportional delays of the form

$$
\begin{aligned}
(u(x) & \left.+a(x) u\left(\gamma_{m} x\right)\right)^{(m)} \\
& =\beta u(x)+\sum_{n=0}^{m-1} b_{n}(x) u^{(n)}\left(\gamma_{n} x\right)+f(x), \quad x \geq 0,
\end{aligned}
$$


with the initial conditions

$$
\sum_{n=0}^{m-1} c_{i n} u^{(n)}(0)=\lambda_{i}, \quad i=0,1, \ldots, m-1,
$$

where $a$ and $b_{n}(n=0,1, \ldots, m-1)$ are given functions, meanwhile, $\beta, \gamma_{n}, c_{i n}$, and $\lambda_{i}$ are constants with $0<\gamma_{n}<1$ $(n=0,1, \ldots, m)$. This class of equations plays an important role in modeling phenomena of the real world.

In the pseudo-spectral methods [25-28], one needs to exactly satisfy the differential equation at specified collocation points in the domain of solution. Generally, the distribution of the collocation nodes can be freely chosen, but an accurate approximations are obtained by selecting the collocation nodes as the zeros of the orthogonal polynomials. For shifted Jacobi polynomials, two commonly used quadrature and collocation nodes, namely, (i) shifted Jacobi-Gauss nodes (in the interior of the domain) and (ii) shifted JacobiGauss-Lobatto nodes (in the interior and at the two endpoints of the domain).

Now, we will present the shifted Jacobi-Gauss-Lobatto type quadratures. Let $x_{N, j}^{(\alpha, \beta)}, 0 \leqslant j \leqslant N$, be the nodes of the Jacobi-Gauss-Lobatto interpolation on $(-1,1)$, and let $\omega_{N, j}^{(\alpha, \beta)}$, $0 \leqslant j \leqslant N$, be the corresponding weights. Throughout this paper, we assume that $x_{L, N, j}, 0 \leqslant j \leqslant N$, stands for the nodes of the shifted Jacobi-Gauss-Lobatto interpolation on the interval $(0, L)$. Thus $x_{L, N, j}^{(\alpha, \beta)}, 0 \leqslant j \leqslant N$, and their corresponding weights are ${\omega_{L, N, j}^{(\alpha, \beta)}}^{(\alpha)}(L / 2)^{\alpha+\beta+1} \omega_{N, j}^{(\alpha, \beta)}, 0 \leqslant j \leqslant$ $N$. Let $S_{N}(0, L)$ be the set of all polynomials of degree $\leq N$. One gets for any $\phi \in S_{2 N-1}(0, L)$,

$$
\begin{aligned}
& \int_{0}^{L}(L-x)^{\alpha} x^{\beta} \phi(x) d x \\
& \quad=\left(\frac{L}{2}\right)^{\alpha+\beta+1} \int_{-1}^{1}(1-x)^{\alpha}(1+x)^{\beta} \phi\left(\frac{L}{2}(x+1)\right) d x \\
& \quad=\left(\frac{L}{2}\right)^{\alpha+\beta+1} \sum_{j=0}^{N} \omega_{N, j}^{(\alpha, \beta)} \phi\left(\frac{L}{2}\left(x_{N, j}^{(\alpha, \beta)}+1\right)\right) \\
& \quad=\sum_{j=0}^{N} \omega_{L, N, j}^{(\alpha, \beta)} \phi\left(x_{L, N, j}^{(\alpha, \beta)}\right) .
\end{aligned}
$$

Let us first introduce some basic notations that will be used in the sequel. We set

$$
S_{N}(0, L)=\operatorname{span}\left\{P_{L, 0}^{(\alpha, \beta)}(x), P_{L, 1}^{(\alpha, \beta)}(x), \ldots, P_{L, N}^{(\alpha, \beta)}(x)\right\} .
$$

The discrete inner product and norm are defined by

$$
\begin{gathered}
(u, v)_{w_{L}^{(\alpha, \beta)}, N}=\sum_{j=0}^{N} u\left(x_{L, N, j}^{(\alpha, \beta)}\right) v\left(x_{L, N, j}^{(\alpha, \beta)}\right) \omega_{L, N, j}^{(\alpha, \beta)}, \\
\|u\|_{w_{L}^{(\alpha, \beta)}, N}=\sqrt{(u, u)_{w_{L}^{(\alpha, \beta)}, N}},
\end{gathered}
$$

where $x_{L, N, j}^{(\alpha, \beta)}$ and $\omega_{L, N, j}^{(\alpha, \beta)}$ are the nodes and the corresponding weights of the shifted Jacobi-Gauss-Lobatto quadrature formula on the interval $(0, L)$, respectively. Obviously,

$$
(u, v)_{w_{L}^{(\alpha, \beta)}, N}=(u, v)_{w_{L}^{(\alpha, \beta)}}, \quad \forall u, v \in S_{2 N-1} .
$$

The shifted Jacobi-Gauss-Lobatto pseudo-spectral method for solving (28) and (29) is to seek $u_{N}(x) \in S_{N}(0, L)$, such that

$$
\begin{aligned}
& \left(u\left(x_{L, N-m, k}^{(\alpha, \beta)}\right)+a\left(x_{L, N-m, k}^{(\alpha, \beta)}\right) u\left(\gamma_{m} x_{L, N-m, k}^{(\alpha, \beta)}\right)\right)^{(m)} \\
& =\beta u\left(x_{L, N-m, k}^{(\alpha, \beta)}\right) \\
& \quad+\sum_{n=0}^{m-1} b_{n}\left(x_{L, N-m, k}^{(\alpha, \beta)}\right) u^{(n)}\left(\gamma_{n} x_{L, N-m, k}^{(\alpha, \beta)}\right) \\
& +f\left(x_{L, N-m, k}^{(\alpha, \beta)}\right), \quad k=0,1, \ldots, N-m, \\
& \quad k=0,1, \ldots, N-m, \\
& \sum_{n=0}^{m-1} c_{i n} u^{(n)}(0)=\lambda_{i}, \quad i=0,1, \ldots, m-1,
\end{aligned}
$$

where the $x_{L, N-m, k}^{(\alpha, \beta)}, k=1,2, \ldots, N-m-1$, are distinct and lie between 0 and $L, x_{L, N-m, 0}^{(\alpha, \beta)}=0$, and $x_{L, N-m, N-m}^{(\alpha, \beta)}=L$. For simplicity in presentation and without loss of generality, assume that $a(x) \equiv 1$. We now derive the collocation algorithm for solving (28) and (29). To do this, consider the solution is approximated by a truncated Jacobi expansion

$$
u_{N}(x)=\sum_{h=0}^{N} a_{h} P_{L, h}^{(\alpha, \beta)}(x), \quad \mathbf{a}=\left(a_{0}, a_{1}, \ldots, a_{N}\right)^{T}
$$

We first approximate $u(x)$ and $u^{n}(x)$ as (32). By substituting these approximation in (28), we get

$$
\begin{aligned}
\sum_{h=0}^{N} a_{h} D^{(m)} P_{L, h}^{(\alpha, \beta)}(x)+\sum_{h=0}^{N} a_{h} D^{(m)} P_{L, h}^{(\alpha, \beta)}\left(\gamma_{m} x\right) \\
=\beta \sum_{h=0}^{N} a_{h} P_{L, h}^{(\alpha, \beta)}(x) \\
\quad+\sum_{n=0}^{m-1} \sum_{h=0}^{N} b_{n}(x) a_{h} D^{(n)} P_{L, h}^{(\alpha, \beta)}\left(\gamma_{n} x\right)+f(x) .
\end{aligned}
$$


Then, by virtue of (8), we deduce that

$$
\begin{aligned}
& \sum_{h=0}^{N} a_{h}\left(\frac{\Gamma(h+\alpha+\beta+m+1)}{L^{n} \Gamma(h+\alpha+\beta+1)} P_{L, h-m}^{(\alpha+m, \beta+m)}(x)\right. \\
& \left.\quad+\gamma_{m}^{m} \frac{\Gamma(h+\alpha+\beta+m+1)}{L^{m} \Gamma(h+\alpha+\beta+1)} P_{L, h-m}^{(\alpha+m, \beta+m)}\left(\gamma_{m} x\right)\right) \\
& =\beta \sum_{h=0}^{N} a_{h} P_{L, h}^{(\alpha, \beta)}(x) \\
& +\sum_{n=0}^{m-1} \sum_{h=0}^{N} \gamma_{n}^{(n)} b_{n}(x) a_{h} \frac{\Gamma(h+\alpha+\beta+n+1)}{L^{n} \Gamma(h+\alpha+\beta+1)} \\
& \quad \times P_{L, h-n}^{(\alpha+n, \beta+n)}\left(\gamma_{n} x\right)+f(x) .
\end{aligned}
$$

Also, by substituting (32) in (29), we obtain

$$
\sum_{n=0}^{m-1} \sum_{h=0}^{N} c_{i n} a_{h} D^{(n)} P_{L, h}^{(\alpha, \beta)}(0)=\lambda_{i}
$$

To find the solution $u_{N}(x)$, we first collocate (20) at the $(N-m+1)$ shifted Jacobi roots that yields

$$
\begin{aligned}
& \sum_{j=0}^{N} a_{j}\left(\frac{\Gamma(j+\alpha+\beta+m+1)}{L^{n} \Gamma(j+\alpha+\beta+1)} P_{L, j-m}^{(\alpha+m, \beta+m)}\left(x_{L, N-m, k}^{(\alpha, \beta)}\right)\right. \\
& +\gamma_{m}^{m} \frac{\Gamma(j+\alpha+\beta+m+1)}{L^{m} \Gamma(j+\alpha+\beta+1)} \\
& \left.\quad \times P_{L, j-m}^{(\alpha+m, \beta+m)}\left(\gamma_{m} x_{L, N-m, k}^{(\alpha, \beta)}\right)\right) \\
& =\beta \sum_{j=0}^{N} a_{j} P_{L, j}^{(\alpha, \beta)}\left(x_{L, N-m, k}^{(\alpha, \beta)}\right) \\
& +\sum_{n=0}^{m-1} \sum_{j=0}^{N} \gamma_{n}^{(n)} b_{n}\left(x_{L, N-m, k}^{(\alpha, \beta)}\right) a_{j} \\
& \quad \times \frac{\Gamma(j+\alpha+\beta+n+1)}{L^{n} \Gamma(j+\alpha+\beta+1)} P_{L, j-n}^{(\alpha+n, \beta+n)}\left(\gamma_{n} x_{L, N-m, k}^{(\alpha, \beta)}\right) \\
& +f\left(x_{L, N-m, k}^{(\alpha, \beta)} \quad k=0,1, \ldots, N-m .\right.
\end{aligned}
$$

Next, (21), after using (7), can be written as

$$
\sum_{n=0}^{m-1} \sum_{j=0}^{N}(-1)^{j-n} c_{i n} a_{j} \frac{\Gamma(j+\beta+1)(j+\alpha+\beta+1)_{n}}{L^{n} \Gamma(j-n+1) \Gamma(n+\beta+1)}=\lambda_{i}
$$

Thus (22) with relation (23) can be written as a linear algebraic system. To do this, let us consider

$$
\begin{gathered}
\mathbf{a}=\left(a_{0}, a_{1}, \ldots, a_{N}\right)^{T}, \\
f_{k}=f\left(x_{L, N-m, k}\right), \quad k=0,1, \ldots, N-m, \\
\mathbf{f}=\left(f_{0}, f_{1}, \ldots, f_{N-m}, \lambda_{0}, \ldots, \lambda_{m-1}\right)^{T} .
\end{gathered}
$$

The matrix system associated with (22) and (23) becomes

$$
\left(A+\gamma_{m}^{m} B+\beta C+\sum_{n=0}^{m-1} \gamma_{n}^{n} D_{n}+E\right) \mathbf{a}=\mathbf{f}
$$

where the matrices $A, B, C, D_{i}, i=1,2, \ldots, m-1$, and $E$ are given explicitly in the following. If we denote that $A=\left(a_{k j}\right)_{0<k, j<N}, B=\left(b_{k j}\right)_{0<k, j<N}, C=\left(c_{k j}\right)_{0<k, j<N}, D_{n}=$ $\left(d_{k j}^{n}\right)_{0<k, j<N}, n=1,2, \ldots, m-1$, and $E=\left(e_{k j}\right)_{0<k, j<N}$, then the elements $a_{k j}, b_{k j}, c_{k j}$, and $d_{k j}^{n}$ are given by

$a_{k j}$

$$
=\left\{\begin{array}{lr}
\frac{\Gamma(j+\alpha+\beta+m+1)}{L^{n} \Gamma(j+\alpha+\beta+1)} & \\
\times P_{L, j-m}^{(\alpha+m, \beta+m)}\left(x_{L, N-m, k}^{(\alpha, \beta)}\right), & k=0,1, \ldots, N-m, \\
0, & j=0,1, \ldots, N, \\
0 & N-m+1, \ldots, N, \\
& j=0,1, \ldots, N,
\end{array}\right.
$$

$b_{k j}$

$$
=\left\{\begin{array}{lr}
\frac{\Gamma(j+\alpha+\beta+m+1)}{L^{m} \Gamma(j+\alpha+\beta+1)} & \\
\times P_{L, j-m}^{(\alpha+m, \beta+m)}\left(\gamma_{m} x_{L, N-m, k}^{(\alpha, \beta)}\right), & k=0,1, \ldots, N-m, \\
& j=0,1, \ldots, N, \\
0, & k=N-m+1, \ldots, N, \\
& j=0,1, \ldots, N,
\end{array}\right.
$$

$c_{k j}$

$$
= \begin{cases}-P_{L, j}^{(\alpha, \beta)} & \\ \times\left(x_{L, N-m, k}^{(\alpha, \beta)}\right), & k=0,1, \ldots, N-m, j=0,1, \ldots, N, \\ 0, & k=N-m+1, \ldots, N, j=0,1, \ldots, N,\end{cases}
$$

$d_{k j}^{n}$

$$
=\left\{\begin{array}{rr}
-b_{n}\left(x_{L, N-m, k}^{(\alpha, \beta)}\right) & \\
\times \frac{\Gamma(j+\alpha+\beta+n+1)}{L^{n} \Gamma(j+\alpha+\beta+1)} & \\
\times P_{L, j-n}^{(\alpha+n, \beta+n)}\left(\gamma_{n} x_{L, N-m, k}^{(\alpha, \beta)}\right), & k=0,1, \ldots, N-m, \\
& j=0,1, \ldots, N, \\
0, & k=N-m+1, \ldots, N, \\
& j=0,1, \ldots, N .
\end{array}\right.
$$


Moreover, the elements of the matrix corresponding to the mixed initial conditions are given by

$$
\begin{aligned}
& e_{k j} \\
& =\left\{\begin{array}{lr}
0, & k=0,1, \ldots, N-m, \\
\sum_{n=0}^{m-1}(-1)^{j-n} & j=0,1, \ldots, N, \\
\times c_{k-N-m+1, n} & \\
\times(j+\beta+1)(j+\alpha+\beta+1)_{n} & \\
\times \frac{k=N-m+1, \ldots, N,}{L^{n} \Gamma(j-n+1) \Gamma(n+\beta+1)} & j=0,1, \ldots, N .
\end{array}\right.
\end{aligned}
$$

In the case of $a(x) \neq 0, b_{n}(x) \neq 0, n=0,1, \ldots, m-1$, and $\beta \neq 0$, the linear system (25) can be solved by forming explicitly the LU factorization; that is, $A+\gamma_{m}^{m} B+\beta C+\sum_{n=0}^{m-1} \gamma_{n}^{n} D_{n}+$ $E=\mathrm{LU}$. The expense of calculating LU factorization is $O\left(N^{3}\right)$ operations, and the expense of solving the linear system (25), provided that the factorization is known, is $O\left(N^{2}\right)$.

\section{Nonlinear High-Order NFDE with Proportional Delay}

In this section, we investigate the shifted Jacobi-GaussLobatto pseudospectral method to numerically approximate the nonlinear high-order NFDE with proportional delay; namely,

$$
\begin{aligned}
& \frac{d^{m} u(x)}{d x^{m}} \\
& =G\left(x, u(x), u\left(\gamma_{0} x\right), \frac{d u\left(\gamma_{1} x\right)}{d x}, \ldots, \frac{d^{m} u\left(\gamma_{m} x\right)}{d x^{m}}\right), \\
& 0 \leq x \leq L,
\end{aligned}
$$

subject to

$$
\sum_{n=0}^{m-1} c_{i n} u^{(n)}(0)=\lambda_{i}, \quad i=0,1, \ldots, m-1,
$$

where $\gamma_{n}, c_{i n}$, and $\lambda_{i}$ are constants with $0<\gamma_{n}<1(n=0$, $1, \ldots, m)$, meanwhile, $G$ is nonlinear in general. This equation is a generalized form of the proportional delay differential equations given in $[14,29,30]$ and plays an important role in modeling phenomena of the real world.

The shifted Jacobi-Gauss-Lobatto pseudospectral approximation for (28) is to find $u_{N}(x) \in S_{N}(0, L)$ such that

$$
\frac{d^{m}}{d x^{m}} u\left(x_{L, N-m, k}^{(\alpha, \beta)}\right)
$$

$$
\begin{aligned}
=G\left(x_{L, N-m, k}^{(\alpha, \beta)}, u\left(x_{L, N-m, k}^{(\alpha, \beta)}\right),\right. & \\
& u\left(\gamma_{0} x_{L, N-m, k}^{(\alpha, \beta)}\right), \frac{d}{d x} u\left(\gamma_{1} x_{L, N-m, k}^{(\alpha, \beta)}\right), \ldots, \\
& \left.\frac{d^{m}}{d x^{m}} u\left(\gamma_{m} x_{L, N-m, k}^{(\alpha, \beta)}\right)\right), \quad k=0,1, \ldots, N-m .
\end{aligned}
$$

Now, we approximate the numerical solution as a truncated series expansion of shifted Jacobi polynomial in the form

$$
u_{N}(x)=\sum_{j=0}^{N} a_{j} P_{L, j}^{(\alpha, \beta)}(x)
$$

Accordingly, (30) can be written as

$$
\begin{aligned}
& \sum_{j=0}^{N} a_{j} \frac{d^{m}}{d x^{m}} P_{L, j}^{(\alpha, \beta)}\left(x_{L, N-m, k}^{(\alpha, \beta)}\right) \\
&=G\left(x, \sum_{j=0}^{N} a_{j} P_{L, j}^{(\alpha, \beta)}\left(x_{L, N-m, k}^{(\alpha, \beta)}\right),\right. \\
& \\
& \sum_{j=0}^{N} a_{j} P_{L, j}^{(\alpha, \beta)}\left(\gamma_{0} x_{L, N-m, k}^{(\alpha, \beta)}\right), \\
& \sum_{j=0}^{N} a_{j} \frac{d}{d x} P_{L, j}^{(\alpha, \beta)}\left(\gamma_{1} x_{L, N-m, k}^{(\alpha, \beta)}\right), \ldots \\
&\left.\sum_{j=0}^{N} a_{j} \frac{d^{m}}{d x^{m}} P_{L, j}^{(\alpha, \beta)}\left(\gamma_{m} x_{L, N-m, k}^{(\alpha, \beta)}\right)\right) \\
& k=0,1, \ldots, N-m .
\end{aligned}
$$

Next, making use of relation (8), thus the high-order derivatives of the proposed solution with proportional delays, $\left(d u\left(\gamma_{1} x\right) / d x\right), \ldots,\left(d^{m} u\left(\gamma_{m} x\right) / d x^{m}\right)$, can be expressed explicitly in terms of the shifted Jacobi polynomials and the expansion coefficients $a_{j}$; consequently

$$
\begin{gathered}
\sum_{j=0}^{N} a_{j} \frac{\Gamma(j+\alpha+\beta+m+1)}{L^{n} \Gamma(j+\alpha+\beta+1)} P_{L, j-m}^{(\alpha+m, \beta+m)}\left(x_{L, N-m, k}^{(\alpha, \beta)}\right) \\
=G\left(x, \sum_{j=0}^{N} a_{j} P_{L, j}^{(\alpha, \beta)}\left(x_{L, N-m, k}^{(\alpha, \beta)}\right), \sum_{j=0}^{N} a_{j} P_{L, j}^{(\alpha, \beta)}\left(\gamma_{0} x_{L, N-m, k}^{(\alpha, \beta)}\right),\right. \\
\sum_{j=0}^{N} a_{j} \gamma_{1}\left(\frac{\Gamma(j+\alpha+\beta+m+1)}{L^{n} \Gamma(j+\alpha+\beta+1)}\right.
\end{gathered}
$$




$$
\begin{array}{r}
\left.\quad \times P_{L, j-m}^{(\alpha+m, \beta+m)}\left(\gamma_{1} x_{L, N-m, k}^{(\alpha, \beta)}\right)\right), \ldots, \\
\sum_{j=0}^{N} a_{j} \gamma_{m}^{m}\left(\frac{\Gamma(j+\alpha+\beta+m+1)}{L^{n} \Gamma(j+\alpha+\beta+1)}\right. \\
\left.\left.\times P_{L, j-m}^{(\alpha+m, \beta+m)}\left(\gamma_{m} x_{L, N-m, k}^{(\alpha, \beta)}\right)\right)\right), \\
k=0,1, \ldots, N-m .
\end{array}
$$

Finally, to find the unknown expansion coefficients $a_{j}, j=$ $0,1, \ldots, N$, we implement any iteration technique to solve a system of $N+1$ nonlinear algebraic equations resulting from the combination of $N-m+1$ nonlinear algebraic equations resulting from (33) and $m$ linear algebraic equations resulting from (29).

\section{Numerical Results}

In this section, we will carry out three test examples to study the validity and effectiveness of the proposed method and also show that high accurate solutions are achieved using a few number of the Jacobi Gauss-Lobatto points. Moreover, comparisons with other methods reveal that the present method is accurate and convenient. All the numerical computations have been performed by the symbolic computation software Mathematica 8.0.

Example 1. Consider the first-order NFDE with proportional delay considered in [31]

$$
\begin{gathered}
u^{\prime}(x)=-u(x)+0.1 u(0.8 x)+0.5 u^{\prime}(0.8 x) \\
+(0.32 x-0.5) e^{-0.8 x}+e^{-x}, \quad x \geq 0, \\
u(0)=0
\end{gathered}
$$

which has the exact solution $x e^{-x}$.

Table 1 lists the absolute error using Jacobi pseudospectral method for three choices of $\alpha, \beta$ at $N=20$ in the interval $[0,1]$. We compare the errors obtained by the proposed method with variational iteration (VI) method [32], the one$\operatorname{leg} \theta$ method $[14,33]$ with $\theta=0.8$, and RKHSM method [31]. The graph of analytical solution and approximate solution in long interval $[0,10]$ for $N=28$ and $\alpha=-1 / 2, \beta=1 / 2$ is displayed in Figure 1 to make it easier to compare with analytical solution. Moreover, in this case the graph of the error is given in Figure 2. Consequently, we conclude that the approximate solution by Jacobi pseudospectral method agree very well with the exact solution.

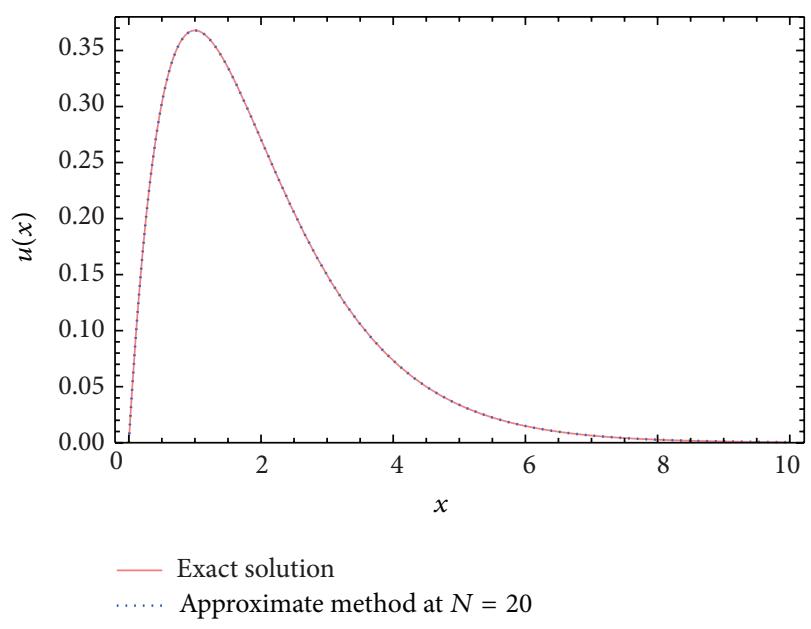

FIgURE 1: Comparison of the approximate solution with the exact solution for $\alpha=-1 / 2, \beta=1 / 2$ at $N=28$ for Example 1 .

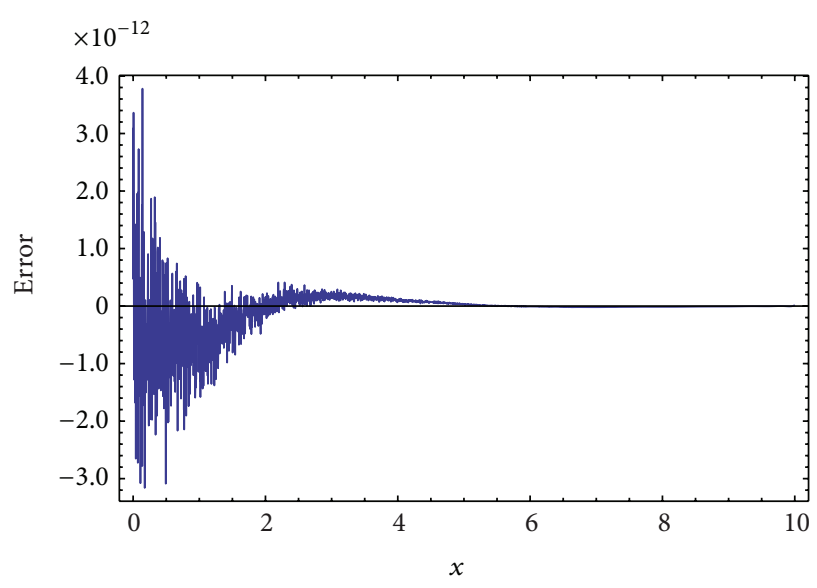

FIGURE 2: The error between the approximate solution and the exact solution in the interval $[0,10]$ for $\alpha=-1 / 2, \beta=1 / 2$ at $N=28$ for Example 1.

Example 2. Let us consider the second-order NFDE with proportional delay

$$
\begin{aligned}
u^{\prime \prime}(x)= & u^{\prime}\left(\frac{x}{2}\right)-\frac{1}{2} x u^{\prime \prime}\left(\frac{x}{2}\right)-\frac{\pi}{2} \\
& \times\left(2 \cos \left(\frac{\pi x}{2}\right)+\pi x \sin \left(\frac{\pi x}{2}\right)\right. \\
& +2 \pi \sin (\pi x)), \quad x \in[0,6], \\
u(0) & =0, \quad u^{\prime}(0)=\pi,
\end{aligned}
$$

which enjoys exact solution $u(x)=\sin (\pi x)$.

In Table 2, we introduce the absolute error using the proposed method at $N=24$ with various choices of $\alpha$ and $\beta$. The resulting graph of (35) for the presented method in the case of $\alpha=\beta=1$ at $N=24$ and the analytic solution are shown in Figure 3. 
TABLE 1: Absolute errors using SJGLP method with various choices of $N, \alpha$, and $\beta$ for Example 1.

\begin{tabular}{|c|c|c|c|c|c|c|c|}
\hline \multirow{2}{*}{$x$} & \multirow{2}{*}{$\alpha$} & \multirow{2}{*}{$\beta$} & \multirow{2}{*}{$\begin{array}{l}\text { SJGLP method } \\
\qquad N=20\end{array}$} & \multirow{2}{*}{ In [31] } & \multicolumn{2}{|c|}{ In [32] } & \multirow{2}{*}{ In [33] } \\
\hline & & & & & $n=5$ & $n=6$ & \\
\hline \multirow{2}{*}{0.1} & $1 / 2$ & $1 / 2$ & $1.58 \cdot 10^{-17}$ & $1.42 \cdot 10^{-4}$ & $2.63 \cdot 10^{-3}$ & $1.30 \cdot 10^{-3}$ & $4.65 \cdot 10^{-3}$ \\
\hline & 0 & 0 & $2.23 \cdot 10^{-17}$ & & & & \\
\hline \multirow{2}{*}{0.2} & $1 / 2$ & $1 / 2$ & $2.90 \cdot 10^{-18}$ & $1.17 \cdot 10^{-4}$ & $4.36 \cdot 10^{-3}$ & $2.14 \cdot 10^{-3}$ & $1.45 \cdot 10^{-2}$ \\
\hline & 0 & 0 & $4.16 \cdot 10^{-17}$ & & & & \\
\hline \multirow{2}{*}{0.3} & $1 / 2$ & $1 / 2$ & $9.21 \cdot 10^{-18}$ & $9.45 \cdot 10^{-4}$ & $5.40 \cdot 10^{-3}$ & $2.63 \cdot 10^{-3}$ & $2.57 \cdot 10^{-2}$ \\
\hline & 0 & 0 & $4.65 \cdot 10^{-17}$ & & & & \\
\hline \multirow{2}{*}{0.4} & $1 / 2$ & $1 / 2$ & $7.09 \cdot 10^{-17}$ & $7.59 \cdot 10^{-4}$ & $5.89 \cdot 10^{-3}$ & $2.84 \cdot 10^{-3}$ & $3.60 \cdot 10^{-2}$ \\
\hline & 0 & 0 & $3.52 \cdot 10^{-17}$ & & & & \\
\hline \multirow{2}{*}{0.5} & $1 / 2$ & $1 / 2$ & $1.94 \cdot 10^{-17}$ & $6.03 \cdot 10^{-4}$ & $5.96 \cdot 10^{-3}$ & $2.83 \cdot 10^{-3}$ & $4.43 \cdot 10^{-2}$ \\
\hline & 0 & 0 & $4.85 \cdot 10^{-17}$ & & & & \\
\hline \multirow{2}{*}{0.6} & $1 / 2$ & $1 / 2$ & $1.68 \cdot 10^{-17}$ & $4.73 \cdot 10^{-4}$ & $5.71 \cdot 10^{-3}$ & $2.67 \cdot 10^{-3}$ & $5.03 \cdot 10^{-2}$ \\
\hline & 0 & 0 & $5.83 \cdot 10^{-17}$ & & & & \\
\hline \multirow{2}{*}{0.7} & $1 / 2$ & $1 / 2$ & $1.22 \cdot 10^{-18}$ & $3.64 \cdot 10^{-4}$ & $5.23 \cdot 10^{-3}$ & $2.39 \cdot 10^{-3}$ & $5.37 \cdot 10^{-2}$ \\
\hline & 0 & 0 & $1.62 \cdot 10^{-17}$ & & & & \\
\hline \multirow{2}{*}{0.8} & $1 / 2$ & $1 / 2$ & $2.42 \cdot 10^{-17}$ & $2.75 \cdot 10^{-4}$ & $4.59 \cdot 10^{-3}$ & $2.04 \cdot 10^{-3}$ & $5.47 \cdot 10^{-2}$ \\
\hline & 0 & 0 & $6.88 \cdot 10^{-17}$ & & & & \\
\hline \multirow{2}{*}{0.9} & $1 / 2$ & $1 / 2$ & $3.93 \cdot 10^{-17}$ & $2.03 \cdot 10^{-4}$ & $3.84 \cdot 10^{-3}$ & $1.64 \cdot 10^{-3}$ & $5.35 \cdot 10^{-2}$ \\
\hline & 0 & 0 & $5.46 \cdot 10^{-18}$ & & & & \\
\hline \multirow{2}{*}{1.0} & $1 / 2$ & $1 / 2$ & $1.33 \cdot 10^{-17}$ & $1.43 \cdot 10^{-4}$ & $3.04 \cdot 10^{-3}$ & $1.22 \cdot 10^{-3}$ & $5.03 \cdot 10^{-2}$ \\
\hline & 0 & 0 & $1.25 \cdot 10^{-16}$ & & & & \\
\hline
\end{tabular}

TABLE 2: Absolute errors using SJGLP method with various choices of $\alpha$ and $\beta$ for Example 2.

\begin{tabular}{lccc}
\hline$x$ & $\alpha=\beta=-1 / 2$ & $\alpha=\beta=1 / 2$ & $\alpha=\beta=0$ \\
\hline 0.0 & $4.13 \cdot 10^{-16}$ & $3.92 \cdot 10^{-16}$ & $1.11 \cdot 10^{-16}$ \\
0.1 & $6.76 \cdot 10^{-10}$ & $1.29 \cdot 10^{-9}$ & $3.57 \cdot 10^{-10}$ \\
0.2 & $5.01 \cdot 10^{-9}$ & $3.70 \cdot 10^{-9}$ & $5.86 \cdot 10^{-10}$ \\
0.3 & $3.24 \cdot 10^{-9}$ & $9.52 \cdot 10^{-9}$ & $1.71 \cdot 10^{-9}$ \\
0.4 & $4.14 \cdot 10^{-8}$ & $5.17 \cdot 10^{-7}$ & $2.16 \cdot 10^{-9}$ \\
0.5 & $2.61 \cdot 10^{-8}$ & $2.88 \cdot 10^{-8}$ & $2.27 \cdot 10^{-9}$ \\
1.0 & $3.72 \cdot 10^{-8}$ & $1.01 \cdot 10^{-8}$ & $2.13 \cdot 10^{-8}$ \\
2.0 & $7.32 \cdot 10^{-8}$ & $3.89 \cdot 10^{-9}$ & $3.59 \cdot 10^{-8}$ \\
3.0 & $2.19 \cdot 10^{-9}$ & $2.84 \cdot 10^{-8}$ & $1.85 \cdot 10^{-7}$ \\
4.0 & $3.52 \cdot 10^{-7}$ & $7.78 \cdot 10^{-7}$ & $3.01 \cdot 10^{-7}$ \\
5.0 & $3.73 \cdot 10^{-6}$ & $1.24 \cdot 10^{-6}$ & $1.80 \cdot 10^{-6}$ \\
6.0 & $3.42 \cdot 10^{-6}$ & $2.97 \cdot 10^{-6}$ & $8.74 \cdot 10^{-6}$ \\
\hline
\end{tabular}

Example 3. Consider the third-order NFDE with proportional delays

$$
\begin{aligned}
& u^{\prime \prime \prime}(x)=(u(x))^{2}+u^{\prime}\left(\frac{x}{2}\right) \\
&+u^{\prime \prime}\left(\frac{x}{3}\right)+\frac{1}{2} u^{\prime \prime \prime}\left(\frac{x}{4}\right)+f(x), \quad x \geq 0, \\
& u(0)=1, \quad u^{\prime}(0)=\frac{1}{3}, \quad u^{\prime \prime}(0)=-\frac{575}{9},
\end{aligned}
$$

where

$$
\begin{aligned}
f(x)=\frac{1}{54} e^{x / 12}( & -13752 \sin (2 x)-54 e^{7 x / 12} \cos ^{2}(8 x) \\
& +1727 \cos (2 x)+6 e^{x / 36} \\
& \times\left(48 \sin \left(\frac{8 x}{3}\right)+575 \cos \left(\frac{8 x}{3}\right)\right) \\
& -18 e^{x / 12}(\cos (4 x)-24 \sin (4 x)) \\
& \left.+2 e^{x / 4}(13752 \sin (8 x)-1727 \cos (8 x))\right) .
\end{aligned}
$$

The exact solution of the problem is $u(x)=e^{x / 3} \cos (8 x)$.

Table 3 lists the maximum absolute errors using SJGLP method in the interval $[0,1]$ at $\alpha=-\beta=0.5$ and different choices of $\alpha$ and $\beta$.

In case of Chebyshev polynomials of the second kind $(\alpha=\beta=1 / 2)$, the graph of exact solution and approximate solution for $N=24$ is plotted in Figure 4 in the interval $[0,3]$.

\section{Conclusion}

In this paper, we have demonstrated the feasibility of SJGLP for solving linear NFDEs with proportional delays. We also have discussed the resulting linear system. Moreover, we have 


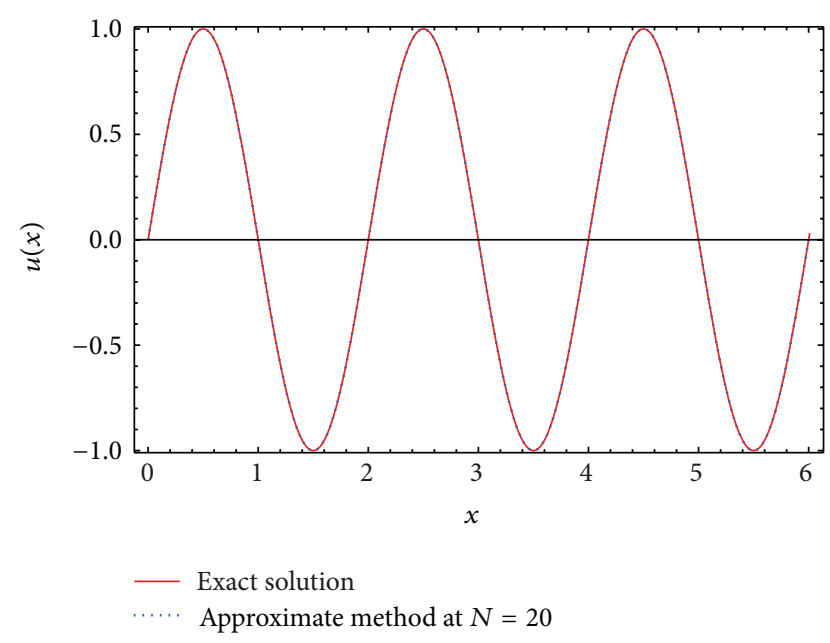

FIGURE 3: Graph of exact solution and approximate solution for $\alpha=$ $\beta=1$ and $N=24$ for Example 2 .

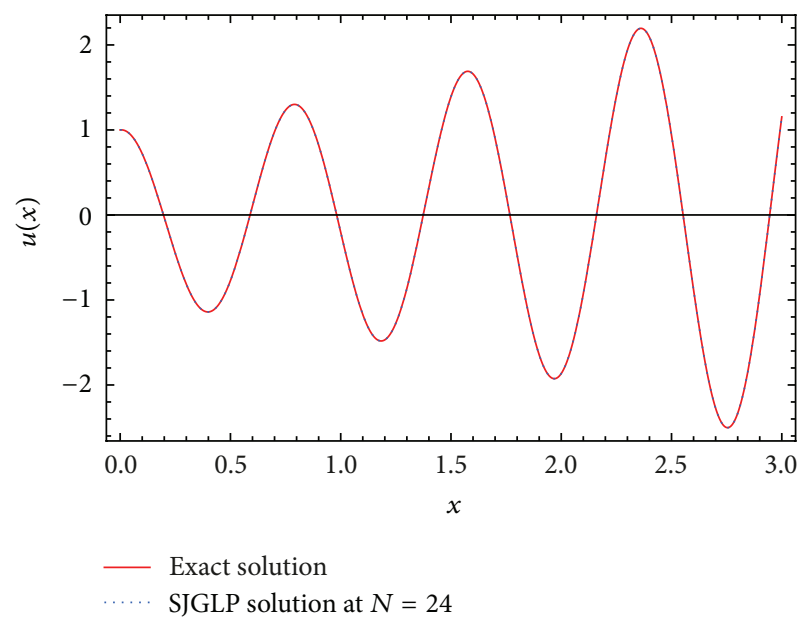

FIgURE 4: Graph of exact solution and approximate solution for $\alpha=$ $\beta=1 / 2$ and $N=24$ for Example 3 .

TABLE 3: Maximum absolute errors using SJGLP method with various choices of $\alpha, \beta$, and $N$ for Example 3 .

\begin{tabular}{lccc}
\hline$t$ & $\alpha=-\beta=0.5$ & $\alpha=-\beta=-0.5$ & $\alpha=\beta=-0.5$ \\
\hline 12 & $1.74 \cdot 10^{-2}$ & $3.86 \cdot 10^{-3}$ & $5.07 \cdot 10^{-3}$ \\
16 & $1.40 \cdot 10^{-6}$ & $2.65 \cdot 10^{-6}$ & $3.54 \cdot 10^{-6}$ \\
20 & $4.38 \cdot 10^{-9}$ & $6.96 \cdot 10^{-10}$ & $9.40 \cdot 10^{-10}$ \\
24 & $8.25 \cdot 10^{-13}$ & $1.63 \cdot 10^{-12}$ & $8.76 \cdot 10^{-13}$ \\
28 & $4.35 \cdot 10^{-13}$ & $3.44 \cdot 10^{-13}$ & $3.22 \cdot 10^{-13}$ \\
\hline
\end{tabular}

implemented the SJGLP method to numerically approximate the nonlinear high-order NFDE with proportional delay.

All the given examples reveal that the results of SJGLP method are in excellent agreement with the analytical solutions. It is concluded from the aforementioned tables and figures that SJGLP method is an accurate and efficient method to solve NFDEs when compared with those generated by some other methods.
In the future work, we address the Jacobi pseudospectral approximation for the solution of linear and nonlinear delay partial differential equations in two and three dimensions (see, e.g., $[17,34])$.

\section{References}

[1] C. Canuto, M. Y. Hussaini, A. Quarteroni, and T. A. Zang, Spectral Methods in Fluid Dynamics, Springer Series in Computational Physics, Springer, New York, NY, USA, 1988.

[2] L. N. Trefethen, Spectral Methods in MATLAB, vol. 10 of Software, Environments, and Tools, SIAM, Philadelphia, Pa, USA, 2000.

[3] E. H. Doha, A. H. Bhrawy, and S. S. Ezz-Eldien, "A new Jacobi operational matrix: an application for solving fractional differential equations," Applied Mathematical Modelling, vol. 36, no. 10, pp. 4931-4943, 2012.

[4] A. H. Bhrawy and M. Al-shomrani, "A shifted Legendre spectral method for fractional order multi-point boundary value problems," Advances in Difference Equations, vol. 2012, article 8, 8 pages, 2012.

[5] A. H. Bhrawy, M. A. Alghamdi, and T. M. Taha, "A new modified generalized Laguerre operational matrix of fractional integration for solving fractional differential equations on the half line," Advances in Difference Equations, vol. 2012, article 179, 12 pages, 2012.

[6] A. Bellen and M. Zennaro, Numerical Methods for Delay Differential Equations, Numerical Mathematics and Scientific Computation, Oxford University Press, New York, NY, USA, 2003.

[7] M. Z. Liu and D. Li, "Properties of analytic solution and numerical solution of multi-pantograph equation," Applied Mathematics and Computation, vol. 155, no. 3, pp. 853-871, 2004.

[8] G. A. Bocharov and F. A. Rihan, "Numerical modelling in biosciences using delay differential equations," Journal of Computational and Applied Mathematics, vol. 125, no. 1-2, pp. 183199, 2000.

[9] M. A. Jafari and A. Aminataei, "Method of successive approximations for solving the multi-pantograph delay equations," General Mathematics Notes, vol. 8, pp. 23-28, 2012.

[10] S. Yüzbaşı, "An efficient algorithm for solving multi-pantograph equation systems," Computers \& Mathematics with Applications, vol. 64, no. 4, pp. 589-603, 2012.

[11] S. Yüzbaşi, N. Şahin, and M. Sezer, "A Bessel collocation method for numerical solution of generalized pantograph equations," Numerical Methods for Partial Differential Equations, vol. 28, no. 4, pp. 1105-1123, 2012.

[12] E. Tohidi, A. H. Bhrawy, and K. Erfani, "A collocation method based on Bernoulli operational matrix for numerical solution of generalized pantograph equation," Applied Mathematical Modelling, vol. 37, no. 6, pp. 4283-4294, 2013.

[13] J. J. Zhao, Y. Xu, H. X. Wang, and M. Z. Liu, "Stability of a class of Runge-Kutta methods for a family of pantograph equations of neutral type," Applied Mathematics and Computation, vol. 181, no. 2, pp. 1170-1181, 2006.

[14] W.-S. Wang and S.-F. Li, "On the one-leg $\theta$-methods for solving nonlinear neutral functional differential equations," Applied Mathematics and Computation, vol. 193, no. 1, pp. 285-301, 2007.

[15] D. Trif, "Direct operatorial tau method for pantograph-type equations," Applied Mathematics and Computation, vol. 219, no. 4, pp. 2194-2203, 2012. 
[16] A. H. Bhrawy, L. M. Assas, E. Tohidi, and M. A. Alghamdi, "Legendre-Gauss collocation method for neutral functionaldifferential equations with proportional delays," Advances in Differential Equations, vol. 2013, article 63, 16 pages, 2013.

[17] Z.-Z. Sun and Z.-B. Zhang, "A linearized compact difference scheme for a class of nonlinear delay partial differential equations," Applied Mathematical Modelling, vol. 37, no. 3, pp. 742752, 2013

[18] L. F. Cordero and R. Escalante, "Segmented tau approximation for test neutral functional differential equations," Applied Mathematics and Computation, vol. 187, no. 2, pp. 725-740, 2007.

[19] M. Muslim, "Approximation of solutions to history-valued neutral functional differential equations," Computers \& Mathematics with Applications, vol. 51, no. 3-4, pp. 537-550, 2006.

[20] O. R. Işik, Z. Güney, and M. Sezer, "Bernstein series solutions of pantograph equations using polynomial interpolation," Journal of Difference Equations and Applications, vol. 18, no. 3, pp. 357374, 2012.

[21] E. K. Ifantis, "An existence theory for functional-differential equations and functional-differential systems," Journal of Differential Equations, vol. 29, no. 1, pp. 86-104, 1978.

[22] A. Iserles and Y. Liu, "On neutral functional-differential equations with proportional delays," Journal of Mathematical Analysis and Applications, vol. 207, no. 1, pp. 73-95, 1997.

[23] C. He, X. Lv, and J. Niu, "A new method based on the RKHSM for solving systems of nonlinear IDDEs with proportional delays," Abstract and Applied Analysis, vol. 2013, Article ID 541935, 13 pages, 2013.

[24] E. Ishiwata, "On the attainable order of collocation methods for the neutral functional-differential equations with proportional delays," Computing, vol. 64, no. 3, pp. 207-222, 2000.

[25] A. H. Bhrawy and A. S. Alofi, "A Jacobi-Gauss collocation method for solving nonlinear Lane-Emden type equations," Communications in Nonlinear Science and Numerical Simulation, vol. 17, no. 1, pp. 62-70, 2012.

[26] A. H. Bhrawy and M. A. Alghamdi, "A shifted Jacobi-GaussLobatto collocation method for solving nonlinear fractional Langevin equation involving two fractional orders in different intervals," Boundary Value Problems, vol. 2012, article 62, 13 pages, 2012.

[27] M. Maleki, I. Hashim, M. Tavassoli Kajani, and S. Abbasbandy, "An adaptive pseudospectral method for fractional order boundary value problems," Abstract and Applied Analysis, vol. 2012, Article ID 381708, 19 pages, 2012.

[28] A. H. Bhrawy, "A Jacobi-Gauss-Lobatto collocation method for solving generalized Fitzhugh-Nagumo equation with timedependent coefficients," Applied Mathematics and Computation, vol. 222, pp. 255-264, 2013.

[29] W. Wang, Y. Zhang, and S. Li, "Stability of continuous RungeKutta-type methods for nonlinear neutral delay-differential equations," Applied Mathematical Modelling, vol. 33, no. 8, pp. 3319-3329, 2009.

[30] J. X. Kuang and Y. H. Cong, Stability of Numerical Methods for Delay Differential Equations, Science Press, Beijing, China, 2005.

[31] X. Lv and Y. Gao, "The RKHSM for solving neutral functionaldifferential equations with proportional delays," Mathematical Methods in the Applied Sciences, vol. 36, no. 6, pp. 642-649, 2013.

[32] X. Chen and L. Wang, "The variational iteration method for solving a neutral functional-differential equation with proportional delays," Computers \& Mathematics with Applications, vol. 59, no. 8, pp. 2696-2702, 2010.
[33] W. Wang, T. Qin, and S. Li, "Stability of one-leg $\theta$-methods for nonlinear neutral differential equations with proportional delay," Applied Mathematics and Computation, vol. 213, no. 1, pp. 177-183, 2009.

[34] Z. Jackiewicz and B. Zubik-Kowal, "Spectral collocation and waveform relaxation methods for nonlinear delay partial differential equations," Applied Numerical Mathematics, vol. 56, no. 3-4, pp. 433-443, 2006. 


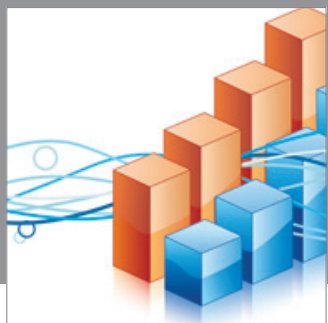

Advances in

Operations Research

mansans

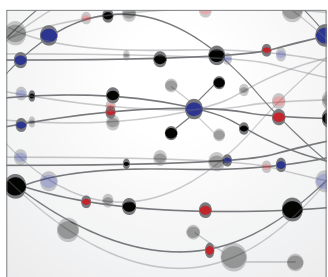

The Scientific World Journal
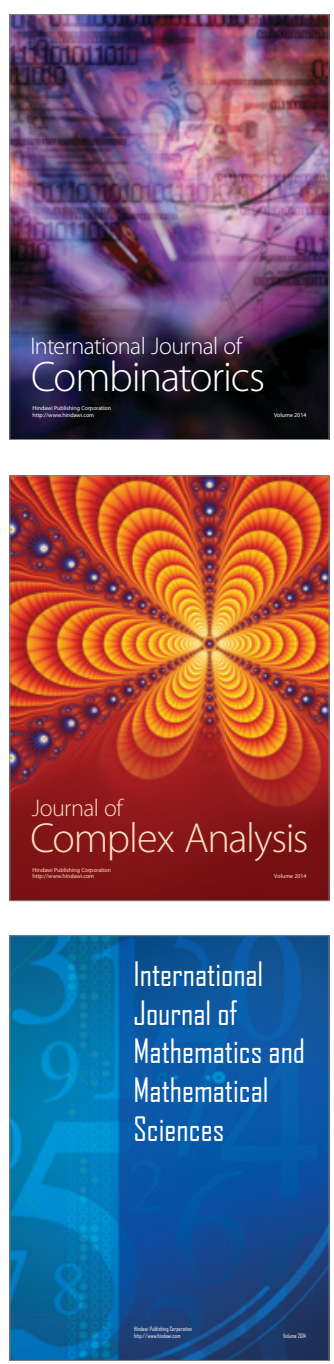
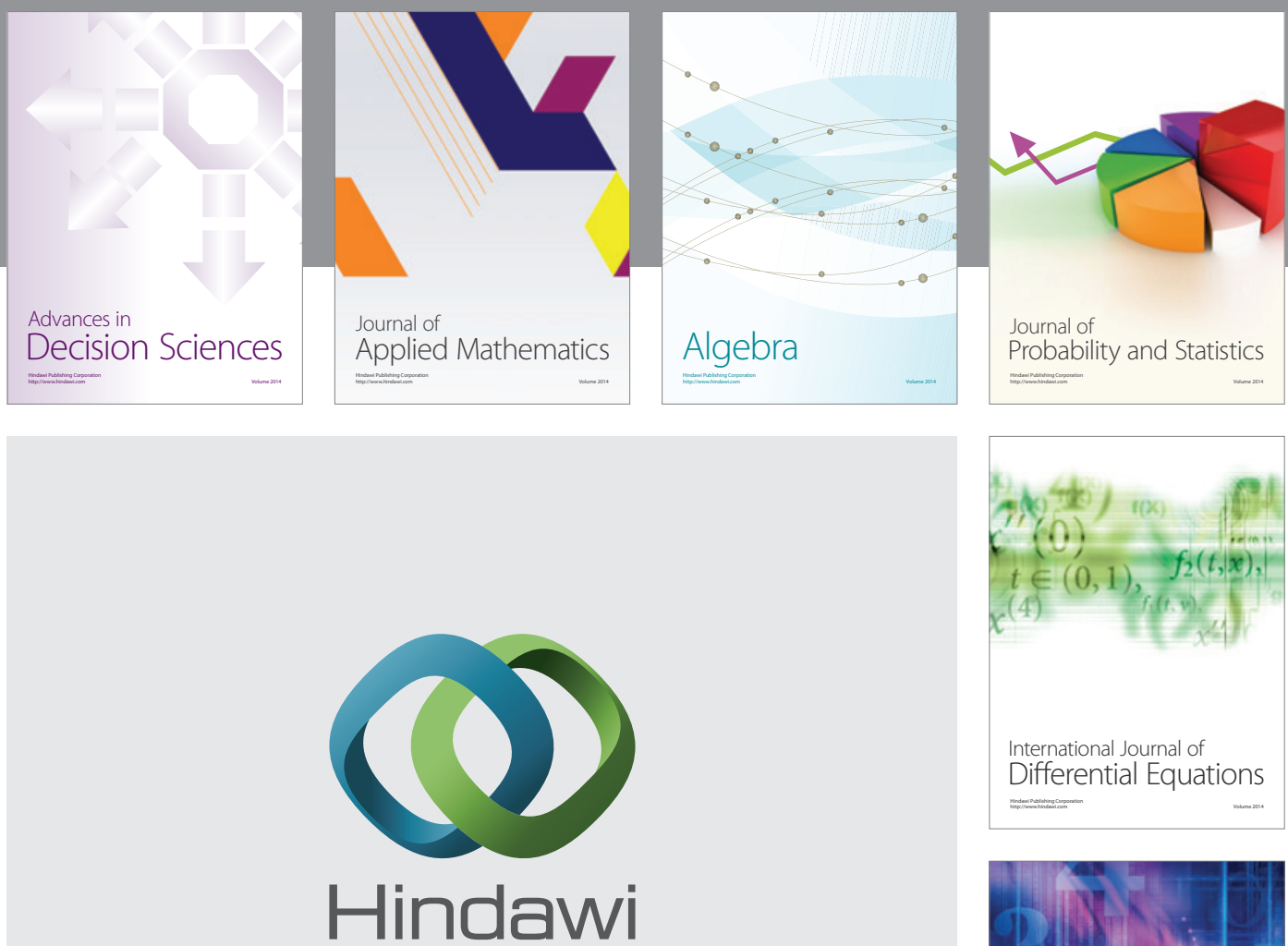

Submit your manuscripts at http://www.hindawi.com
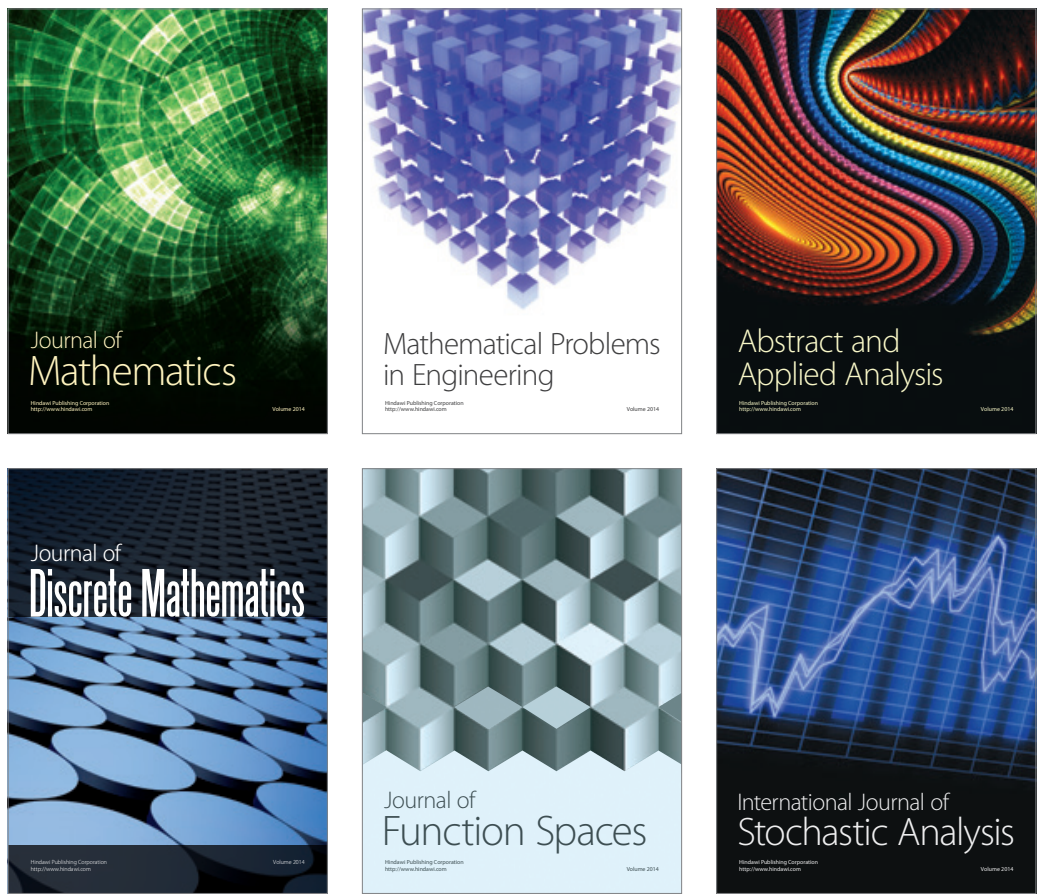

Journal of

Function Spaces

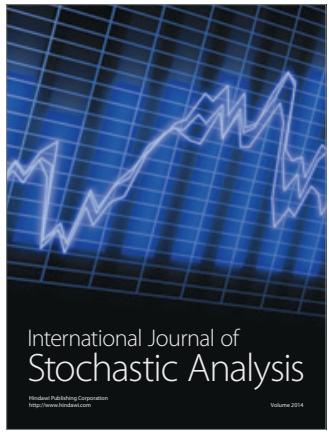

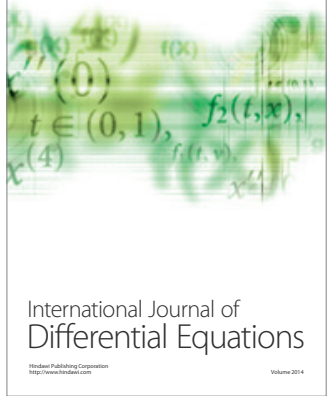
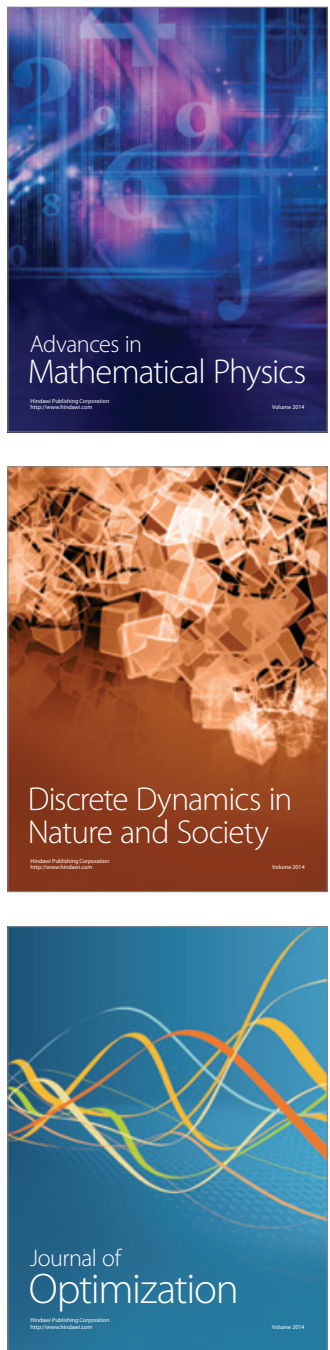